\title{
Triple-pomeron dynamics: valence vs. glue
}

\author{
V.R. Zoller ${ }^{\mathrm{a}}$ \\ aTEP, Moscow 117218, Russia
}

Three pomerons are known to couple via the gluon loop and interactions of this kind which are responsible for high-mass diffraction are described in terms of the gluonic structure function of the QCD pomeron. We show that the triple-pomeron coupling via the light quark loop associated with the "valence" in the pomeron is of the same strength as the purely gluonic coupling. The large $Q^{2}$ behavior of this new contribution is described by the DLLA evolution from the non-perturbative $f \bar{f}$ valence state of the pomeron. Numerical estimates of the high-mass diffraction structure functions based on the consistent account of both couplings are in good agreement with experimental data.

In this communication we discuss the new triple-pomeron ${ }^{1}$ contribution to the high-mass diffractive deep inelastic scattering (DIS) which comes from excitation of the $(q \bar{q})(f \bar{f})$ Fock states of the photon,

$\gamma^{*}+p \rightarrow(q \bar{q})(f \bar{f})+p$

where $q$ and $f$ are the light quarks [2]. Compared to the purely gluonic triple pomeron interactions which scale is determined by the correlation radius of non-perturbative gluons, $R_{c} \sim 0.25$ fermi [3], the $f \bar{f}$-pair in the quark loop connectig pomerons has a large non-perturbative size, $r_{f} \simeq$ $1 / m_{f} \sim 1$ fermi, and enters the evolution of sea of the pomeron in precisely the same way as the valence quark density enters the evolution of the sea of nucleons. We demonstrate that such a pQCD evolution holds at least to the DLLA accuracy. We show that although the $(q \bar{q})(f \bar{f})$ contribution is of higher order in pQCD coupling $\alpha_{S}$ it is enhanced by a potentially large numerical factor, $\propto\left[\sigma\left(r_{f}\right) / \sigma\left(R_{c}\right)\right]^{2}$, where $\sigma(r) \sim r^{2}$ is the color dipole cross section, and numerically it is comparable to the leading order gluonic triple-pomeron term.

The process (11) represents the first step of $\left(\beta, Q^{2}\right)$-evolution of the diffractive structure function from the $f \bar{f}$ "valence" quark component of the pomeron. Its space-time picture in the lab. reference frame is as follows. The high-energy

${ }^{1}$ for definitions and early analysis see [1] photon converts into the colorless $q \bar{q}$ pair at large distances upstream the target. The $q \bar{q}$ dipole with transverse separation $\mathbf{r}$ acts like a source of gluons. Emitted gluon has a transverse coordinate $\boldsymbol{\rho}$ and the momentum fraction $z_{g}$ which are distributed in accord with the wave function of the $q \bar{q} g$ state 4 . This gluon has a virtuality $\kappa^{2} \simeq-\kappa^{2}$ and in its turn converts into the pair of quarks $f \bar{f}$ of size $\mathbf{R}$. To isolate the leading terms of the 4-parton interaction cross section one has to look into the impact parameter structure of the diffractive $(q \bar{q})(f \bar{f})$ state. The analysis of ref. [5] shows that the effective size of the quark-anti-quark component of the photon is generally process-dependent. In particular, the proton structure function $F_{2 p}\left(x, Q^{2}\right)$ in the scaling limit is dominated by the small-size $(q \bar{q})$ fluctuations, $r^{2} \sim 1 / Q^{2}$. The case of the diffraction dissociation (DD) of the photon is quite different. The $q \bar{q}$ fluctuations of the size $1 / Q^{2}$ are altogether negligible and the DD structure function is dominated by the $q \bar{q}$ states of the largest possible size $R \sim 1 / m_{q} \sim 1$ fermi. Thus, there are two different scattering regimes and two very different scales. Both of them are important for the process (11) where the two quark pairs, $q \bar{q}$ and $f \bar{f}$, are in the regimes of inclusive DIS and DD, respectively. As we are interested in the large mass excitations, $M^{2} \propto Q^{2} / z_{g}$, the Sudakov variable for the gluon is $z_{g} \ll 1$. If so, the small size $q \bar{q}$-dipole and radiated gluon $g$ are separated 
from the origin (which is fixed by the projectile photon) by the distances $z_{g} \rho$ and $\left(1-z_{g}\right) \rho$, respectively. The $f \bar{f}$-pair produced by the gluon with virtuality $\kappa^{2} \gtrsim R_{c}^{2}$ is in the DD regime and, as such, is very asymmetric. Its Sudakov variable is $\alpha \lesssim m_{f}^{2} / \kappa^{2}$. Two conclusions can be drawn immediately. First, the size of $f \bar{f}$-pair is large, $R \sim 1 / m_{f}$, and, second, the $f$-(anti)quark is separated from the origin by the large distance $\sim R\left(1-m_{f}^{2} / \kappa^{2}\right)$, while the other (anti)quark goes along the parent gluon with small separation $\sim R m_{f}^{2} / \kappa^{2}$. Hence, the DLLA ordering of sizes 6] which vary along the partonic ladder from very large $R \sim 1 / m_{f}$ in the bottom $f \bar{f}$-cell of the ladder down to very small $r \sim 1 / \sqrt{Q^{2}}$ in the top, $q \bar{q}$ - cell,

$r \ll \rho \ll R$.

As a result the 4-parton system $(q \bar{q})(f \bar{f})$ acts like a triplet-anti-triplet dipole of size $R \sim 1 / m_{f} \sim 1$ fermi and as such has large interaction cross section $\sigma_{(q \bar{q})(f \bar{f})} \simeq \sigma(R)$. The distribution of $f \bar{f}$ color dipoles in the gluon of transverse momentum $\boldsymbol{\kappa}$ is identical to that in the photon subject to the substitutions $N_{c} \alpha_{e m} e_{f}^{2} \rightarrow T_{F} \alpha_{S}\left(\kappa^{2}\right)$ and $Q^{2} \rightarrow \kappa^{2}$, so that the diffractive cross section of interest equals

$$
\begin{aligned}
& \left.\left(Q^{2}+M^{2}\right) \frac{d \sigma^{D}((q \bar{q})(f \bar{f}))}{d t d M^{2}}\right|_{t=0}=\frac{1}{16 \pi} \int d^{2} \boldsymbol{\kappa} \\
& \times \frac{d g_{q \bar{q}}\left(Q^{2}, \boldsymbol{\kappa}\right)}{d^{2} \boldsymbol{\kappa}} \frac{T_{F} \alpha_{S}\left(\boldsymbol{\kappa}^{2}\right)}{N_{c} \alpha_{e m} e_{f}^{2}}\left\langle f \bar{f}\left|\sigma^{2}\left(x_{\mathbf{I P}}, \mathbf{R}\right)\right| f \bar{f}\right\rangle,
\end{aligned}
$$

where the flux of gluons in the parent $q \bar{q}$ state is

$$
\begin{aligned}
\frac{d g_{q \bar{q}}\left(Q^{2}, \boldsymbol{\kappa}\right)}{d^{2} \boldsymbol{\kappa}} & =\int_{0}^{1} d z_{q} \int d^{2} \mathbf{r}\left|\Psi_{\gamma^{*}}\left(Q^{2}, z_{q}, \mathbf{r}\right)\right|^{2} \\
\times & \frac{2 e_{q}^{2} C_{F} \alpha_{S}\left(r^{2}\right)}{\pi^{2}} \cdot \frac{[1-\exp (i \boldsymbol{\kappa} \mathbf{r})]}{\left(\boldsymbol{\kappa}^{2}+\mu_{G}^{2}\right)^{2}} \boldsymbol{\kappa}^{2} .
\end{aligned}
$$

Finally, notice that

$$
\begin{aligned}
& \frac{\boldsymbol{\kappa}^{2}}{4 \pi^{2} \alpha_{e m}} \frac{1}{16 \pi e_{f}^{2}}\left\langle f \bar{f}\left|\sigma^{2}\left(x_{\mathbf{I P}}, \mathbf{R}\right)\right| f \bar{f}\right\rangle \\
& =\left.\frac{\boldsymbol{\kappa}^{2}}{4 \pi^{2} \alpha_{e m}} \frac{d \sigma\left(\gamma^{*}\left(\boldsymbol{\kappa}^{2}\right) \rightarrow f \bar{f}\right)}{d t}\right|_{t=0}
\end{aligned}
$$

$=\frac{1}{e_{f}^{2}} \int_{0}^{1} \frac{d \beta}{\beta} f_{f \bar{f}}^{D}\left(t=0, x_{\mathbf{I P}}, \beta, \boldsymbol{\kappa}^{2}\right)=N_{f \bar{f}}^{\mathbf{P}}\left(x_{\mathbf{I P}}, \boldsymbol{\kappa}^{2}\right)$

where $N_{f \bar{f}}^{\mathbf{I P}}\left(x_{\mathbf{I P}}, \boldsymbol{\kappa}^{2}\right)$ can be reinterpreted as a number of charged valence partons, i.e., twice the number of $f \bar{f}$ dipoles, in the pomeron. Upon the substitution of (5) and (4) into (3) one readily recovers the dipole representation

$\left.\left(Q^{2}+M^{2}\right) \frac{d \sigma_{q \bar{q} g}^{D}}{d t d M^{2}}\right|_{t=0}=\left\langle q \bar{q}\left|\sigma^{\mathbb{P}}\left(x_{\mathbf{I P}}, \mathbf{r}\right)\right| q \bar{q}\right\rangle$,

in which $\sigma^{\mathbb{P}}\left(x_{\mathbf{I P}}, \mathbf{r}\right)$ is evaluated for the unintegrated gluon structure function evolved from the $f \bar{f}$ state of the pomeron $\mathcal{F}_{f \bar{f}}^{\mathbf{P}_{\mathbf{P}}}\left(\beta, \boldsymbol{\kappa}^{2}\right)=\left(C_{F} \alpha_{S} / \pi\right) N_{f \bar{f}}^{\mathbf{P}_{\mathbf{P}}}\left(x_{\mathbf{I P}}, \boldsymbol{\kappa}^{2}\right)$. Furthermore, $N_{f \bar{f}}^{\mathbf{I P}}\left(x_{\mathbf{I P}}, \boldsymbol{\kappa}^{2}\right)$ vanishes at $\boldsymbol{\kappa}^{2}=0$ and, according to [5], flattens at $\kappa^{2} \gg m_{f}^{2}$. The DLLA analysis of $q \bar{q} g_{1} \ldots g_{n}$ excitation developed in 4 can readily be extended to the higher, $(q \bar{q}) g_{1} . . g_{n}(f \bar{f})$, states. The numerical results for

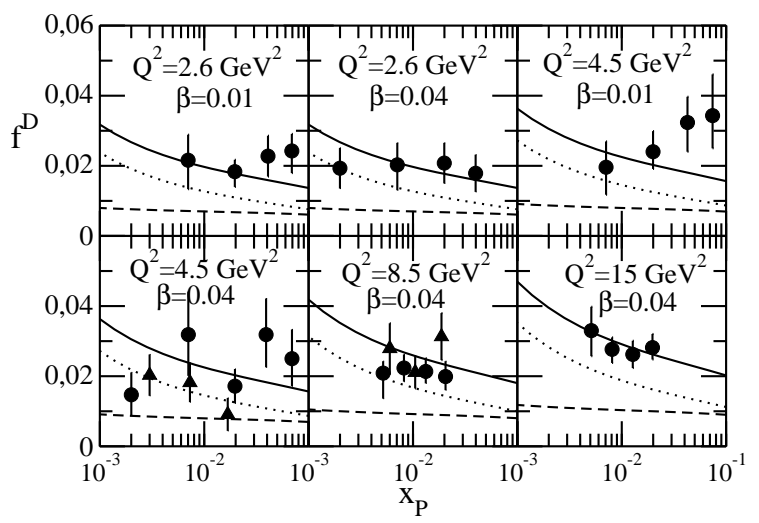

Figure 1. The comparison with the experimental data on small- $\beta$, small- $x_{\mathbf{I P}}$ diffractive structure function (7), full circles; 8, full triangle) of the theoretical evaluation of $f^{D(3)}=f_{q \bar{q} g}^{D(3)}+f_{(q \bar{q})(f \bar{f})}^{D(3)}$ shown by the solid line. The dotted line corresponds to $f_{q \bar{q} g}^{D(3)}$ and the dashed line represents $f_{(q \bar{q})(f \bar{f})}^{D(3)}$. 
high-mass, small- $\beta$, diffraction depend on the input dipole cross section $\sigma(x, \mathbf{r})$. Here we evaluate the lowest order $q \bar{q} g$ [3] and $(q \bar{q})(f \bar{f})$ [2] contributions to diffractive DIS

$$
\begin{aligned}
f^{D(4)}(t= & \left.0, x_{\mathbf{I P}}, \beta, Q^{2}\right)=\frac{Q^{2}}{4 \pi^{2} \alpha_{e m}} \\
& \times\left.\left(Q^{2}+M^{2}\right) \frac{d \sigma^{D}}{d t d M^{2}}\right|_{t=0}
\end{aligned}
$$

in a specific color dipole BFKL model 9] which gives a good description of the proton structure function data. The applicability domain of the small- $\beta$, small- $x_{\mathbf{I P}}$ formalism is $\beta, x_{\mathbf{I P}}<$ $x_{0} \ll 1$, the experience with inclusive DIS suggests $x_{0} \sim 0.03$, although the theoretical curves in fig. 1 are stretched up to $x_{\mathbb{P}}=0.1$. This small- $\beta$, small- $x_{\mathbf{I P}}$ domain is almost at the boundary of the HERA experiments, the corresponding experimental data on the $t$-integrated diffractive structure function $f^{D(3)}\left(x_{\mathbf{I P}}, \beta, Q^{2}\right)$ from $\mathrm{H} 1$ (7], circles) and ZEUS ([8, triangles) are shown in fig. 1. We evaluate this structure function as $f^{D(3)}\left(x_{\mathbf{I P}}, \beta, Q^{2}\right)=\int d t f^{D(4)}\left(t, x_{\mathbf{I P}}, \beta, Q^{2}\right) \approx$ $\frac{1}{B_{3 \mathbb{P}}} f^{D(4)}\left(t=0, x_{\mathbf{I P}}, \beta, Q^{2}\right)$ with the central value of the diffraction slope $B_{\mathbb{I P}}=B_{D}=7.2 \pm 1.1_{-0.9}^{+0.7}$ $\mathrm{GeV}^{-2}$ as reported by ZEUS 10. The apparent growth of the experimentally observed $f^{D(3)}\left(x_{\mathbf{I P}}, \beta, Q^{2}\right)$ towards large $x_{\mathbf{I P}} \sim 0.1$ is usually attributed to the non-vacuum admixture to the pomeron exchange. Two features of the theoretical results for small- $\beta$ diffraction are noteworthy. First, the contributions from $q \bar{q} g$ and higherorder $(q \bar{q})(f \bar{f})$ states are of comparable magnitude because $R_{c} \ll r_{f}$ and the latter is enhanced $\propto\left[\sigma\left(x_{\mathbf{P}}, r_{f}\right) / \sigma\left(x_{\mathbf{I P}}, R_{c}\right)\right]^{2}$. Second, because of the same inequality of the important dipole sizes, $R_{c} \ll r_{f}$, the $x_{\mathbf{I P}}$-dependence of the $q \bar{q} g$ excitation is steeper than that of the $(q \bar{q})(f \bar{f})$ excitation, the numerically significant contribution from the $(q \bar{q})(f \bar{f})$ excitation makes the overall $x_{\mathbf{I P}}$-dependence of $f^{D(3)}\left(x_{\mathbf{I P}}, \beta, Q^{2}\right)$ weaker than evaluated in for the pure $q \bar{q} g$ excitation. The solid curve in fig. 1 is the combined contribution from the two mechanisms. It is in reasonable agreement with the HERA data.

It is a pleasure to thank Roberto Fiore for his kind invitation and warm hospitality at the
Diffraction 2004. This work has been partly supported by the INTAS grant 00-00366 and the DFG grant 436RUS17/101/04.

\section{REFERENCES}

1. K.A. Ter-Martirosyan, Phys. Lett. B44 (1973) 179; A.B.Kaidalov and K.A.TerMartirosyan, Nucl. Phys. B75 (1974) 471; G. Ingelman and P. E. Schlein, Phys. Lett. B152 (1985) 256.

2. N.N. Nikolaev, W. Schaefer, B.G. Zakharov and V.R. Zoller, JETP Letter 80 (2004) 423, hep-ph/0408113

3. M. Genovese, N.N. Nikolaev, B.G. Zakharov J. Exp. Theor. Phys. 81 (1995) 633.

4. N.N. Nikolaev and B.G. Zakharov, J. Exp. Theor. Phys. 78 (1994) 598 Z. Phys. C64 (1994) 631.

5. N.N. Nikolaev and B.G. Zakharov, Z. Phys. C53 (1992) 331.

6. V. N. Gribov and L. N. Lipatov, Sov. J. Nucl. Phys. 15 (1972) 438; Y. L. Dokshitzer, Sov. Phys. JETP 46 (1977) 641; G. Altarelli and G. Parisi, Nucl. Phys. B126 298 (1977).

7. H1 Collab., Measurements of the Diffractive Structure Function $F_{2}^{D(3)}$ at HERA, submitted to XX Int. Symp. on Lepton and Photon Interactions, July 23, 2001, Rome; Z. Phys. C76 (1997) 613.

8. ZEUS Collab., J. Breitweg et al., Eur. Phys. J. C6 (1999) 43.

9. N.N. Nikolaev, B.G. Zakharov and V.R. Zoller, JETP Lett. 66 (1997) 138; N.N.Nikolaev, J. Speth and V.R.Zoller, Phys. Lett. B473 (2000) 157; J. Exp. Theor. Phys. 93 (2001) 957; V.R. Zoller, Phys. Lett. B509 (2001) 69.

10. ZEUS Collab., Eur. Phys. J. C1 (1998) 81. 